\title{
Isolated periaortitis in a 53-year-old male
}

\author{
Rakul Nambiar $^{1 *}$, Kripesh Kannoth ${ }^{1}$ and Dalus Dae ${ }^{1}$ \\ ${ }^{1}$ Department of Internal Medicine, Government Medical College Hospital, Trivandrum, India
}

\begin{abstract}
Chronic periaortitis includes a specific group of rare diseases which is characterized by the presence of abnormal fibroinflammatory tissue in the periaortic retroperitoneum. We describe a case of nonaneurysmal form of chronic periaortitis, recognized as isolated periaortitis, in a male patient who presented with low back pain. We report this case to highlight the fact that periaortitis can present as an isolated primary condition and can be reported without aortic aneurysm or retroperitoneal fibrosis.
\end{abstract}

\section{Case report}

A 53-year-old male presented with low backache of 3 month's duration. The pain was of moderate intensity, unaffected by motion, and not relieved by bed-rest. He had lost approximately nine kilograms over the last 6 months. He did not give any history of fever. Physical examination revealed blood pressure of $200 / 110 \mathrm{~mm} \mathrm{Hg}$ in upper limbs and $180 / 110 \mathrm{~mm} \mathrm{Hg}$ in both lower limbs. The remainder of physical examination was essentially normal. Blood investigations revealed elevated erythrocyte sedimentation rate of $130 \mathrm{~mm}$ and elevated $\mathrm{C}$-reactive protein. Renal and liver function tests were essentially normal. Autoimmune (ANA, ANCA) and infectious work-up (serological testing for syphilis) were negative. Chest and lumbar spine radiographs were normal. A computerized tomography (CT) scan of the lumbar spine was undertaken to exclude any inflammatory process which showed retroperitoneal soft tissue thickening. Hence, CT angiography of aorta (Figure 1) was done which revealed extensive abdominal aortic thickening without any abnormal dilatation. In addition, there was a retroperitoneal concentric periaortic soft tissue mass (rind of periaortic tissue) causing luminal narrowing of the abdominal aorta

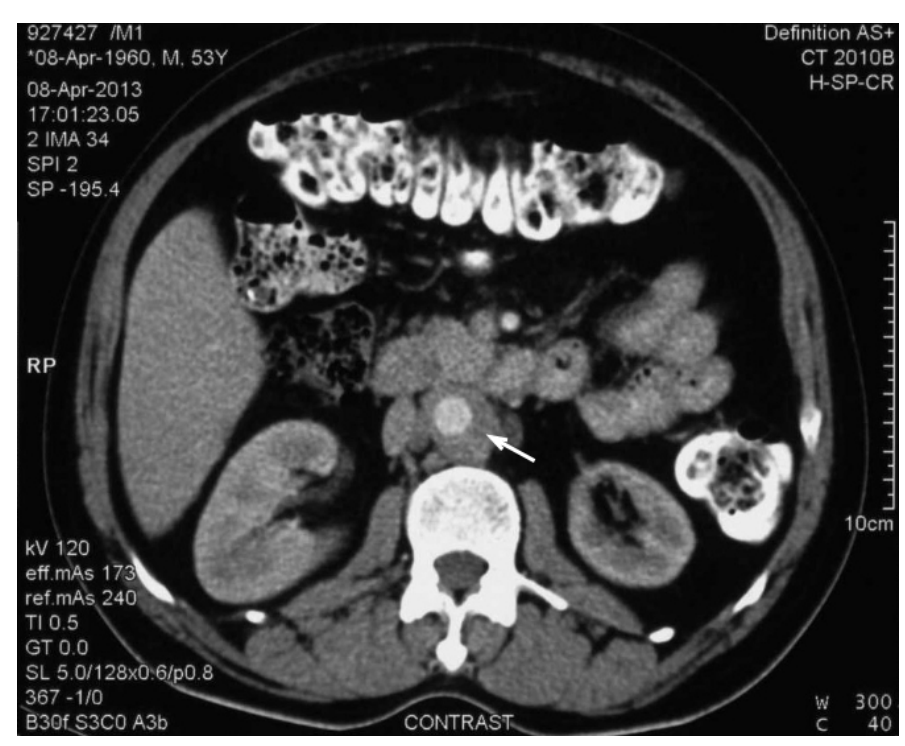

Figure 1. CT abdomen revealed extensive aortic thickening without any abnormal dilatation and significant ostial stenosis of the left renal artery. Infectious workup for vasculitis was also negative. There was no ureteric obstruction or hydronephrosis. This presentation was consistent with the diagnosis of isolated periaortitis confined to abdominal aorta. He was started on antihypertensives and steroids (prednisone $60 \mathrm{mg}$ daily). On follow-up after 12 months, his blood pressure was controlled (130/80 mm Hg). Repeated CT scans showed progressive resolution of the periaortic rind with no evidence of abdominal aorta aneurysm.

\section{Discussion}

Chronic periaortitis includes a variety of conditions like idiopathic retroperitoneal fibrosis, perianeurysmal retroperitoneal fibrosis and inflammatory abdominal aortic aneurysm [1-3]. These conditions have similar clinical and histopathological findings. Most patients with periaortitis suffer from constitutional symptoms and have elevated acute-phase reactant levels, positive autoantibodies and, in few cases, have autoimmune diseases affecting other organs [4]. In view of the absence of any systemic autoimmune disease and the negative autoimmune and infectious work-up, the pathogenesis of the disease in our case may be the result of a local reaction to advanced atherosclerosis. Biopsy is necessary to exclude other disease such as malignancy or infections. In our patient, biopsy was not performed because of the periaortic location of the mass. A similar case of nonaneurysmal form of chronic periaortitis has been described in a 53-year-old man by Zeina et al. [5]. This patient also presented with chronic low back pain. He was initially treated with steroid and later methotrexate was added to help steroid tapering. Like our case this patient too had progressive resolution of the periaortic rind with treatment.

Isolated periaortitis represents the nonaneurysmal form of chronic periaortitis and usually involves the abdominal aorta (particularly the infra-renal portion). This disease primarily causes stenosis of the major branches of the abdominal aorta like celiac trunk, superior

${ }^{*}$ Correspondence to: Rakul Nambiar, Internal Medicine Resident, Department of Internal Medicine, Government Medical College Hospital, Trivandrum, India, Tel: +919633464816

Key words: vasculitis, isolated periaortitis, systemic hypertension

Received: March 10, 2017; Accepted: April 19, 2017; Published: April 22, 2017 
mesenteric or renal arteries as reported in the present case (renal artery), rather than entrapment of the retroperitoneal structures like ureters as in the idiopathic retroperitoneal fibrosis manifestation. The classical presentation of isolated periaortitis includes constitutional symptoms like pain, fever, weight loss, and anemia. Renovascular hypertension may occur secondary to renal artery stenosis. Depending on involvement of other vessels, claudication, and other manifestations of vascular impairment may accompany this disorder. Chronic periaortitis has been hypothesized to be a consequence autoantibodies reacting against antigens like oxidized low-density lipoproteins and ceroid in atherosclerotic plaque [6-8].

Contrast-enhanced CT, magnetic resonance imaging, and positron emission tomography (PET) scan are the imaging modalities of choice for isolated periaortitis. PET scan will demonstrate periaortic activity and can thus be helpful in identifying the extent of involvement [9[. PET will also detect occult infections or malignancies and can assess the status of sdisease activity [5].

Steroids are usually effective in chronic periaortitis and induce remission of the clinical symptoms, normalization of the acutephase reaction, reduction in size of the retroperitoneal mass and also resolution of the obstructive complications $[9,10]$. Several different immunosuppressive drugs have been used as steroid-sparing agents or in patients not responding to steroids alone or when steroids cannot be tapered. Our patient responded to steroids and did not developing retroperitoneal fibrosis or abdominal aorta aneurysm during the follow-up period of 12 months.

\section{References}

1. Parums DV (1990) The spectrum of chronic periaortitis. Histopathology 16: 423-431. [Crossref]

2. Vaglio A, Buzio C (2005) Chronic periaortitis: a spectrum of disease CurrOpinRheumatol17:34-40. [Crossref]

3. Mitnick H, Jacobowitz G, Krinsky G, Eberle M, Rosenzweig B, et al. (2004) Periaortitis: gadolinium-enhanced magnetic resonance imaging and response to therapy in four patients. Ann VascSurg18: 100-107. [Crossref]

4. Carels T, Verbeken E, Blockmans D (2005) p-ANCA-associated periaortitis with histological proof of Wegener's granulomatosis: case report. ClinRheumatol24: 83-86. [Crossref]

5. Zeina AR, Gleb S, Naschitz JE, Loberman Z, Barmeir E (2007) Isolated periaortitis: Clinical and imaging characteristics. Vasc Health and Risk Manag 3:1083-1086. [Crossref]

6. Mitchinson MJ (1970) The pathology of idiopathic retroperitoneal fibrosis. J ClinPathol 23:6819. [Crossref]

7. Parums D, Mitchinson MJ (1981) Demonstration of immunoglobulin in the neighbourhood of advanced atherosclerotic plaques. Atherosclerosis 38: 211-216. [Crossref]

8. Parums DV, Brown DL, Mitchinson MJ (1990) Serum antibodies to oxidized lowdensity lipoprotein and ceroid in chronic periaortitis. Arch Pathol Lab Med 114: 383387. [Crossref]

9. Treglia G, Mattoli MV, Leccisotti L, Ferraccioli G, Giordano A (2011) Usefulness of whole-body fluorine-18-fluorodeoxyglucose positron emission tomography in patients with large-vessel vasculitis: a systematic review. Clin Rheum 30: 1265-1275. [Crossref]

10. Kardar AH, Kattan S, Lindstedt E, Hanash K (2002) Steroid therapy for idiopathic retroperitoneal fibrosis: dose and duration. J Urol168: 550-555. [Crossref]

11. Jois RN, Gaffney K, Marshall T, Scott DG (2004) Chronic periaortitis. Rheumatology (Oxford) 43: 1441-1446. [Crossref]

Copyright: (C2017 Nambiar R. This is an open-access article distributed under the terms of the Creative Commons Attribution License, which permits unrestricted use, distribution, and reproduction in any medium, provided the original author and source are credited. 